Proom, H. \& WoIwod, A. J. (1951). J. gen. Microbiol. 5, 930-938.

\title{
Amine Production in the Genus Proteus
}

\author{
By H. PROOM ANd A. J. WOIWOD \\ The Wellcome Research Laboratories, (Biological Division), Beckenham, Kent
}

SUMMARY: All of 43 strains of Proteus vulgaris, 75 strains of $P$. mirabilis and 10 strains of $P$. morganii when grown in an acid-hydrolysed casein or nutrient broth medium produced isobutylamine and two other amines tentatively identified as isoamylamine and $\beta$-methylbutylamine. These amines were not produced by any of 239 strains representative of other species in the family Enterobacteriaceae grown under the same conditions. This character is sufficiently constant to be included in the generic description. Ten strains of $P$. rettgeri did not produce these amines, and for this and other reasons it is proposed that this species be excluded from the genus Proteus.

The extension of the genus Proteus by Rauss (1936) to include the relatively slow urea-decomposing species $P$. morganii and by Rustigian \& Stuart (1943) to include the anaerogenic mannitol-fermenting species $P$. rettgeri increases the difficulty of distinguishing between Proteus spp. and non-lactose-fermenting strains which are probably more closely related to other genera. A test specific for Proteus spp. therefore would be of value in defining this genus. We have shown (Proom \& Woiwod, 1949) that when Proteus spp. are grown in an acid-hydrolysed casein medium a characteristic change occurs on the developed chromatograms of the cultures. This change consists in the appearance of two ninhydrin-positive spots which have $R_{F}$ values greater than leucine on the single dimensional chromatogram using $n$-butanol+acetic acid as the solvent. We suggested that these ninhydrin-positive substances were polypeptides. We have confirmed that on acid 'hydrolysis' these substances produce amino-acids, but the deduction that therefore they were polypeptides was incorrect. As will be demonstrated, these substances are amines and amine production is characteristic of the genus Proteus.

\section{EXPERIMENTAL}

\section{Amine production by Proteus}

When counter-current extraction methods were used in an attempt to isolate from Proteus cultures the two new substances which appeared on the developed chromatogram these substances were often lost when solutions containing the separated fractions were evaporated to dryness. This suggested the substances were volatile, and it was found that they could be separated from the culture filtrates by steam distillation at alkaline reaction.

A strain of Proteus was grown on an acid-hydrolysed casein medium (Proom \& Woiwod, 1949). Seven litres of culture filtrate were adjusted to pH 9.0 and steam-distilled into $0.1 \mathrm{~N}-\mathrm{HCl}$, and the distillate dried on a waterbath in a vacuum. The material was made alkaline and extracted with ether, and the ether was removed by heating on a water-bath. The weight of the 
dried residue was $1.5 \mathrm{~g}$. This was dissolved in $10 \mathrm{ml}$. distilled water saturated with $n$-butanol and distributed between $n$-butanol and distilled water in an all-glass 27-unit Craig separator (Craig \& Post, 1949). The distribution was studied by paper chromatography. The two components were almost completely separated after one hundred transfers. The respective solutions containing the two separated components were separately bulked and evaporated to dryness in a vacuum. The yields were approximately $130 \mathrm{mg}$. of the slower running material and $350 \mathrm{mg}$. of the faster running material.

The slower running substance proved to be isobutylamine. It was compared

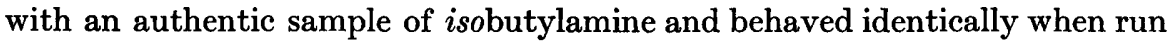
on chromatograms using several solvents. The oxalate derivative was prepared and it had the same melting-point ( $167^{\circ}$ uncorr.) as the oxalate prepared from the authentic isobutylamine. The mixed melting-point of the two derivatives was not depressed. This amine is probably derived from valine.

A number of Proteus strains were grown on a basal medium containing inorganic salts, ammonium chloride and glucose with nicotinic and pantothenic acids added as essential nutrients. Under these conditions the amines were not produced. The simplest medium in which Proteus spp. regularly produced only the slower running substance was the basal medium to which glutamic acid and valine had been added. In a few instances a little of the slower running substance was produced by the addition of valine only. No amines were produced by the addition of glutamic acid only.

The faster running substance was not completely identified but seems likely to be a mixture of isoamylamine and $\beta$-methylbutylamine produced from leucine and isoleucine respectively. The isolated fraction was compared chromatographically with an authentic sample of isoamylamine and could not be distinguished from it with any of the several solvents used. Strains of Proteus spp. grown on the previously described basal medium with added glutamic acid and leucine or isoleucine produced only the faster running substance. The intensity of the faster running spots on the developed chromatograms from cultures grown on the medium with added isoleucine was much less than those from the medium with added leucine, suggesting that the major constituent of the faster running substance was derived from leucine. A sample of $\beta$-methylbutylamine was not available, but it seems unlikely that it could be easily distinguished from isoamylamine by chromatographic methods. Attempts to characterize the faster running material by the preparation of derivatives failed and we attribute this failure to the probability that the material was a mixture of these two amines. The amine nature of this material is evident from the fact that it is volatile at alkaline reaction and gives a positive ninhydrin reaction. The chromatographic evidence strongly supports the view that it is a mixture of isoamylamine and $\beta$-methylbutylamine produced from leucine and isoleucine respectively.

Both the slower and faster running materials were eluted from paper chromatograms and acid hydrolysed. The hydrolysate was examined chromatographically, and a number of ninhydrin-positive spots were obtained. The observation reported previously (Woiwod \& Proom, 1950) was confirmed, but 
there can be little doubt that these two substances are amines and not polypeptides as was assumed from the hydrolysis experiments. The mechanisms responsible for these reactions are not known. This is the second time we have observed unexpected chemical reactions occurring under these conditions, the first instance being the production of a number of substances from $\gamma$-aminobutyric acid (Woiwod \& Proom, 1950).

It was found that after drying a mixture of glucose and isobutylamine or glucose and isoamylamine and chromatographing the dissolved residue, strong ninhydrin-positive reactions were obtained in positions corresponding to valine and leucine respectively. It is possible that carbohydrate material eluted from the paper is in part responsible for the observed effects. It is clear that the appearance of fresh ninhydrin-positive substances after treatment with hydrochloric acid cannot be taken as unequivocal evidence of the presence of peptides. All our experiments were controlled by the hydrolysis of eluates from blank paper, and the observed effects could not be attributed to peptides originally present in the paper.

\section{Chromatographic analysis of Proteus cultures}

Strains were grown for 3 days at $37^{\circ}$ in an acid-hydrolysed casein medium (Proom \& Woiwod, 1949), the medium being dispensed in $2 \mathrm{ml}$. amounts in 1 oz. screw-capped bottles. After incubation the bottles were steamed for $30 \mathrm{~min}$. and the cultures examined by the chromatographic technique described by Woiwod (1949).

The chromatograms of the cultures of all the 128 strains of Proteus examined showed the characteristic ninhydrin-positive spots. Amine production was therefore demonstrated in all the Proteus cultures. Of these strains 43 were $\boldsymbol{P}$. vulgaris, $75 P$. mirabilis and $10 P$. morganii. These strains had been isolated from a variety of sources and covered a wide range of serological types.

We also examined 50 strains of each of the genera Salmonella, Shigella and Escherichia, 25 strains of Aerobacter, 9 strains of Klebsiella, 7 strains of Serratia, 6 strains of Erwinia, 40 strains of uncertain taxonomic status, including many paracolon types, and 10 strains of $P$. rettgeri, some of which were received from the National Collection of Type Cultures (N.C.T.C. 7475-81), two from this collection under the name of Bacterium kandiense (N.C.T.C. 3162, 3163) and one fresh isolate. In no instance was the production of amines observed by the chromatographic technique.

\section{Ninhydrin paper test for the presence of amines}

Since primary amines are volatile at alkaline reaction and react with ninhydrin at room temperature to give a purple colour, it was possible to develop a simple test for the presence of amines which is suitable for routine bacteriological work. After considerable preliminary investigation the following conditions were found to be suitable for the test.

The cultures are grown for $24 \mathrm{hr}$. at $37^{\circ}$ in $4 \mathrm{oz}$. flat screw-capped bottles (United Glass Bottle Co.) containing $20 \mathrm{ml}$. nutrient broth. A sheet of What- 
man no. 54 filter-paper is soaked in a $0.5 \%(\mathrm{w} / \mathrm{v})$ solution of ninhydrin in $n$-butanol and dried at room temperature for $15 \mathrm{~min}$. An approximate $1.5 \mathrm{~cm}$. square is cut from the paper and placed between an extra rubber liner and the bottle cap, both liner and cap being perforated centrally with a hole about $0.5 \mathrm{~cm}$. diameter. The cap is removed from the culture bottle and three drops of capryl alcohol, $2 \mathrm{ml}$. $10 \mathrm{~N}-\mathrm{NaOH}$ and about $0.5 \mathrm{~g}$. aluminium powder (British Drug Houses Ltd., coarse, grease-free) is added. The cap containing the ninhydrin paper is then screwed on the culture bottle which is left at room temperature. The bottle is gently shaken to produce a vigorous flow of hydrogen. The hydrogen carries the amines through the filter-paper where they react with ninhydrin to give a purple colour. This colour develops at room temperature, usually within 15 min., and reaches maximum intensity within $1 \mathrm{hr}$. With broth controls and cultures which do not produce amines the filterpaper remains white. Some cultures produce a considerable amount of ammonia and this may react with ninhydrin to give a yellow colour, but this change is seldom observed within the first $15 \mathrm{~min}$. The intensity of the purple colour varies with the strain, but all the 128 strains of Proteus tested under these conditions gave an unequivocal purple within 15 min. All of 50 strains representative of other species in the family Enterobacteriaceae gave a negative reaction. Although it is unnecessary to remove the cap in order to read the test, the purple colour appears very much more intense when viewed by transmitted light.

We found it necessary to adhere fairly closely to this technique. When the concentration of ninhydrin used for the test paper is decreased much below $0.5 \%$ the test becomes insensitive, and when the concentration is raised above $1.0 \%$ the controls may show a reddish tinge. The $n$-butanol solution of ninhydrin keeps well, but papers should be freshly prepared for each test. When this test is performed with smaller quantities of culture, i.e. $5 \mathrm{ml}$. in $1 \mathrm{oz}$. screw-capped bottles, the amount of amine produced by some strains is not sufficient to give a good positive result. Sufficient space must be left between the surface of the culture and the cap since, in spite of the capryl alcohol, considerable frothing occurs, and if froth reaches the ninhydrin paper, false positive reactions occur. In spite of the considerable evolution of hydrogen no trouble was experienced with spray reaching the filter-paper and thus giving false positive reactions. No advantage is gained by developing the colour by heating the cap in a hot air oven; some test papers develop a purplish tinge on prolonged heating. When a number of cultures are being examined the caps may be removed and the capryl alcohol and alkali added to a row of bottles, but after adding the aluminium powder the test caps must be screwed on within a few secounds, otherwise considerable loss of amine occurs.

\section{Nutritional requirements of Proteus rettgeri}

Since all our ten strains of $\boldsymbol{P}$. rettgeri failed to produce amines it was necessary to reconsider the taxonomy of this species, and it was felt that nutritional studies would be of value. The nutritional requirements of these strains were determined by the methods described by Knight \& Proom (1950). 
All 10 strains grew in a medium in which ammonia and a number of synthetic amino-acids were the only source of nitrogen and without the addition of growth factors.

It is well established that a requirement for nicotinic acid is a characteristic property of Proteus spp. Fildes (1938) first demonstrated this requirement for $P$. vulgaris, and Pelczar \& Porter $(1940 a)$ for $P$. morganii. The latter organism also requires pantothenic acid as an essential nutrient. Quite large numbers of strains have been examined; for example, Pelczar \& Porter $(1940 b)$ examined 169 strains of $P$. vulgaris and $P$. mirabilis, and only 5 of these strains did not require nicotinic acid.

To check our methods and as additional confirmation of the well-established observations we examined 76 strains of Proteus, including representatives of the species vulgaris, mirabilis and morganii. It was considered justifiable for this purpose not to distinguish between the requirements for nicotinic acid and for pantothenic acid. The strains were therefore grown on two media, one containing hydrolysed casein and without growth factors and a second similar medium with added nicotinic and pantothenic acids. Of the 76 strains 73 failed to grow on the hydrolysed casein medium but grew on this medium with added nicotinic and pantothenic acids. Of the three anomalous strains one required the growth factors when the hydrolysed casein was replaced by a mixture of seven amino-acids (asparagine, alanine, glutamic acid, leucine, methionine, proline and serine) and the remaining two required the growth factors when the hydrolysed casein was replaced by ammonia.

\section{Biochemical reactions}

The more important diagnostic biochemical reactions of our Proteus strains were determined. The fermentation reactions were determined in $\frac{1}{4}$ oz. screwcapped bottles containing $c .3 \mathrm{ml}$. of $1.0 \%$ peptone water $+0.5 \%$ of the relevant carbohydrate, with bromocresolpurple as indicator. $\mathbf{H}_{2} \mathrm{~S}$ production was determined by growth in $10 \mathrm{ml}$. nutrient broth in cotton-wool plugged test-tubes with a strip of lead acetate paper as the indicator. Indole production was determined by Kovács's (1928) method, using amyl alcohol as the solvent. Urea decomposition was determined by Christensen's (1946) method. Amine production was determined by both the chromatographic and the ninhydrin test-paper methods.

The results of the biochemical tests are summarized in Table 1. These tests were performed primarily to check the identity and purity of our cultures. The strains have been classified according to Bergey's Manual (1948), since this appears to be the simplest classification. Those strains which failed to ferment sucrose or to produce indole were classified as $\boldsymbol{P}$. mirabilis, although a number of such strains were received as $\boldsymbol{P}$. morganii. $\mathrm{H}_{2} \mathrm{~S}$ production was marked and occurred within $24 \mathrm{hr}$. with the species vulgaris, mirabilis and morganii, but was weak and slow with rettgeri, taking 4 days. There were a few deviations from the typical patterns for each species. A number of strains were tested for gelatin liquefaction, primarily to confirm that this character is variable within the group. Of the 46 strains of $\boldsymbol{P}$. vulgaris or $\boldsymbol{P}$. mirabilis examined, one 
strain of $\boldsymbol{P}$. vulgaris and five of $\boldsymbol{P}$. mirabilis failed to liquefy gelatin, the rate of liquefaction being variable and showing little correlation with species. All 10 strains of $P$. morganii failed to liquefy gelatin.

Table 1. Biochemical reactions of Proteus species

\begin{tabular}{|c|c|c|c|c|c|c|c|c|c|c|}
\hline \multirow[b]{2}{*}{ Species } & \multirow{2}{*}{$\begin{array}{l}\text { No. of } \\
\text { strains } \\
\text { tested }\end{array}$} & \multicolumn{5}{|c|}{ Fermentation of } & \multicolumn{3}{|c|}{ Production of } & \multirow{2}{*}{$\begin{array}{c}\text { Urea } \\
\text { decomp. }\end{array}$} \\
\hline & & Glucose & Lactose & Mannitol & Maltose & Sucrose & Indole & $\mathbf{H}_{2} \mathrm{~S}$ & Amines & \\
\hline$P$. vulgaris & 43 & AG & - & - & AG & AG & + & + & + & $+(1)$ \\
\hline P. mirabilis & 75 & AG & - & - & - & $\begin{array}{l}\text { AG late } \\
\text { or - }\end{array}$ & - & + & 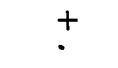 & $+(2)$ \\
\hline P. morganii & 10 & AG & - & $-(1)$ & - & - & + & + & + & + \\
\hline P. rettgeri & 10 & A* & - & A & - & $\begin{array}{c}\text { A slow } \\
\text { or - }\end{array}$ & + & \pm & - & + \\
\hline
\end{tabular}

$\mathbf{A G}=$ acid and gas; $\mathbf{A}=$ acid $;+=$ positive $;-=$ negative .

The bracketed figures give the numbers of strains which gave anomalous reactions.

* One strain of $P$. rettgeri produced a little gas from glucose.

\section{DISCUSSION}

The genus Proteus represents a fairly well-defined group within the peritrichously flagellated Gram-negative rods. Hauser (1885) first described the genus, noting the characteristic swarming on agar plates. He named two species, $\boldsymbol{P}$. vulgaris and $\boldsymbol{P}$. mirabilis, which were distinguished by speed of gelatin liquefaction. Smith (1894) described the fermentation by Proteus spp. of glucose and sucrose with production of small volumes of gas, and the failure to ferment lactose. These reactions were regarded as characteristic of the genus. Wenner \& Rettger (1919) divided their cultures into two species: $P$. vulgaris which fermented maltose and $P$. mirabilis which did not. Moltke (1927) demonstrated that his cultures of Proteus rapidly hydrolysed urea. Rauss (1936) examined a number of cultures of Morgan's bacillus no. 1, and on biochemical and cultural grounds included this species in the genus Proteus. This inclusion is now generally accepted. Rustigian \& Stuart (1943), from a study of 48 strains of Bacterium rettgeri (Hadley, Elkins \& Caldwell, 1918) concluded that this species should be included in the genus Proteus.

Bergey's Manual (1948) divides the genus into four species, $P$. vulgaris, $P$. mirabilis, $\boldsymbol{P}$. morganii and $\boldsymbol{P}$. rettgeri, on the basis of fermentation of maltose, sucrose and mannitol and the production of indole. Topley \& Wilson's Principles (1946) gives the failure to ferment mannitol and ability to produce $\mathrm{H}_{2} \mathrm{~S}$ as generic characters, and divides the genus into a number of subgroups on the basis of fermentation of maltose and sucrose, indole production, rate of hydrolysis of gelatin and serum, and peptonization of milk. Specific names are not ascribed to these subgroups nor is there reference to the species rettgeri.

Fulton (1943) examined 18 strains of Bacterium columbense (Castellani), and suggested that these organisms should be classified together with Morgan's bacillus no. 1 into a new genus Morganella. Our examination of six strains of 
Bact. columbense received from the National Collection of Type Cultures (708, $4647,2702,5254,5497,5498$ ) is in agreement with the description given by Fulton. We consider this species a paracolon organism; apart from its failure to ferment ractose it closely resembles Escherichia coli. None of these six strains of Bact. columbense produced amines. We think that no useful purpose would be served by creating a separate genus for Morgan's bacillus and that this would obscure the close relationship which exists between this species and other members of the genus Proteus.

Rustigian \& Stuart $(1943,1945)$ stated that $P$. morganii and $P$. rettgeri do not produce $\mathrm{H}_{2} \mathrm{~S}$ and the inability to produce $\mathrm{H}_{2} \mathrm{~S}$ is included in the description of these two species given in Bergey's Manual (1948). Rustigian \& Stuart (1945) appreciated that many authors have reported $\mathrm{H}_{2} \mathrm{~S}$ production by $P$. morganii and suggested that this discrepancy was due to the methods used and the interpretation of the results. They did not describe the method they used. We have found that with the lead acetate test-paper technique $P$. morganii is an active producer of $\mathrm{H}_{2} \mathrm{~S}$ and $P$. rettgeri a feeble producer of $\mathrm{H}_{2} \mathrm{~S}$ and we are of opinion that $\mathrm{H}_{2} \mathrm{~S}$ production is a characteristic property of both the genus Proteus and the species $P$. morganii.

We have shown that the ability to produce amines distinguishes $P$. vulgaris, $\boldsymbol{P}$. mirabilis and $\boldsymbol{P}$. morganii from other species in the family Enterobacteriaceae and we feel that this character is sufficiently constant and characteristic to be included in the genic description. The classification of the species rettgeri, does however, require reconsideration. Rustigian \& Stuart (1943) placed this organism in the genus Proteus for the reason that it has fermentation reactions similar to those of other Proteus spp. except that it is anaerogenic and ferments mannitol; it is an active producer of urease and under suitable conditions may swarm in a manner reminiscent of $\boldsymbol{P}$. morganii. The evidence against the classification of rettgeri in the genus Proteus would now appear to be strong. The species is anaerogenic, ferments mannitol, does not produce amines and does not require nicotinic acid as an essential nutrient.

We consider this evidence sufficient to exclude rettgeri from the genus Proteus. If rettgeri be included it follows that with non-lactose-fermenting strains of Enterobacteriaceae inclusion in the genus Proteus depends virtually on the single qualitative character of marked decomposition of urea. Although techniques for urease production, such as those described by Rustigian \& Stuart (1941) and Christensen (1946), effectively differentiate between the marked decomposition of urea characteristic of Proteus species and of rettgeri on the one hand, and the less active decomposition of urea by other species such as Aerobacter, nevertheless this difference is still only quantitative. Moreover, an occasional strain of Proteus fails to decompose urea. On the other hand, if our subsequent definition of the genus Proteus be accepted, then the classification depends on a number of characters, i.e. the fermentation of glucose with small gas volumes, failure to ferment lactose or mannitol, marked decomposition of urea, production of $\mathrm{H}_{2} \mathrm{~S}$, production of amines and a nutritional requirement for nicotinic acid. The genus then represents a well-defined and clearly recognizable group of organisms. We do not propose to suggest an 
alternative classification of Bact. rettgeri, since this is part of the problem of the classification of the family Enterobacteriaceae as a whole, and is outside the scope of this paper.

The proposed description of the genus Proteus is a modification of that given by Borman, Stuart \& Wheeler (1945). The modification consists of including failure to ferment mannitol, production of $\mathrm{H}_{2} \mathrm{~S}$, production of amines and a requirement for nicotinic acid as generic characters. The definition is as follows :

Gram-negative, non-sporing rods. Pleomorphic in young cultures. Usually actively motile at $25^{\circ}$; motility may be weak at $37^{\circ}$. Flagella peritrichous when present. Aerobic, poor growth when grown under anaerobic conditions. Motile forms may produce characteristic swarming on nutrient agar. Ferments glucose, but not lactose or mannitol, with the production of small volumes of gas. Decomposition of urea, marked. $\mathrm{H}_{2} \mathrm{~S}$ produced. Amines produced. Nicotinic acid is required as an essential nutrient.

\section{Type species-Proteus vulgaris (Hauser)}

We are indebted to Prof. B. C. J. G. Knight for help with the nutritional studies, to Mr E. Harris for technical assistance, and to Drs A. A. Miles and F. Kauffmann for most of the strains of Proteus examined.

\section{REFERENCES}

Bergey's Manual of Determinative Bacteriology (1948). 6th ed. Ed. Breed, R. S., Murray, E. G. D. \& Hitchens, A. P. London: Baillière, Tindall and Cox.

Borman, E. L., Stuart, C. A. \& Wheeler, K. M. (1944). Taxonomy of the family Enterobacteriaceae. J. Bact. 48, 351.

Christensen, W. B. (1946). Urea decomposition as a means of differentiating Proteus and paracolon organisms from each other and from Salmonella and Shigella types. J. Bact. 52, 461.

Craig, L. C. \& Post, O. (1949). Apparatus for countercurrent distribution. Anal. Chem. 21, 500.

Fildes, P. (1938). The growth of Proteus on ammonium lactate plus nicotinic acid. Brit. J. exp. Path. 19, 239.

Fulton, M. (1943). The identity of Bacterium columbense Castellani. J. Bact. 46, 79.

Hadley, P., Elkins, M. W. \& Caldwell, D. W. (1918). Bull. Agric. Exp. Sta. Rhode Island College, no. 174.

Hauser, G. (1885). UUber Fäulnisbakterien, Leipzig. From Topley \& Wilson's Principles of Bacteriology and Immunology (1946), 3rd ed. p. 652.

Knight, B. C. J. G. \& Proom, H. (1950). A comparative survey of the nutrition and physiology of mesophilic species in the genus Bacillus. J. gen. Microbiol. 4, 508.

Kovács, N. (1928). Eine vereinfachte Methode zum Nachweis der Indolbildung durch Bakterien. Z. ImmunForsch. 55, 311.

Moltke, O. (1927). Contributions to the Characterization and Systematic Classification of Bac. proteus vulgaris (Hauser). Copenhagen: Levine and Munksgaard.

Pelczar, M. J. \& Porter, R. J. (1940a). Pantothenic acid and nicotinic acid as essential growth substances for Morgan's bacillus (Proteus morganii). Proc. Soc. exp. Biol., N.Y., 43, 151.

Pelczar, M. J. \& Porter, R. J. (1940b). The utilization of nicotinic acid and related pyridine compounds by the Proteus groups of organisms. J. Bact. 39, 429 .

Proom, H. \& Worwod, A. J. (1949). The examination by partition paper chromatography of the nitrogen metabolism of bacteria. J. gen. Microbiol. 3, 319. 
Rauss, K. F. (1936). The systematic position of Morgan's bacillus. J. Path. Bact. $42,183$.

Rustigian, R. \& Stuant, C. A. (1941). Decomposition of urea by Proteus. Proc. Soc. exp. Biol., N.Y., 47, 108.

Rustigian, R. \& Stuart, C. A. (1943). Taxonomic relationships in the genus Proteus. Proc. Soc. exp. Biol., N.Y., 53, 241.

Rustigian, R. \& StUart, C. A. (1945). The biochemical and serological relationships of organisms of the genus Proteus. J. Bact. 49, 419.

Smith, T. (1894). Modification, temporary and permanent, of the physiological characters of bacteria in mixed cultures. Trans. Ass. Amer. Phys. 9, 85.

Topley \& Wilson's Principles of Bacteriology and Immunity (1946). 3rd ed. Ed. Wilson, G. S. \& Miles, A. A. London: Edward Arnold and Co.

Wenner, J. J. \& Rettger, L. F. (1919). A systematic study of the Proteus group of bacteria. J. Bact. 4, 331.

Worwon, A. J. (1949). A technique for examining large numbers of bacterial culture filtrates by partition chromatography. J. gen. Microbiol. 3, 312.

Worwod, A. J. \& Proom, H. (1950). Identification of characteristic extracellular ninhydrin-positive substances produced by some bacteria. J.gen. Microbiol. 4, 501.

(Received 5 May 1951) 